\title{
RETOS Y REALIDADES DEL DOCENTE DE PRIMARIA EN EL USO DE LA TECNOLOGÍA COMO HERRAMIENTA DE ENSEÑANZA EN TIEMPOS DE CONTINGENCIA
}

\section{CHALLENGES AND REALITIES OF ELEMENTARY SCHOOL TEACHERS IN THE USE OF TECHNOLOGY AS A TEACHING TOOL IN CONTINGENCY TIMES}

Lizeth Armenta-Zazueta ${ }^{1}$ * (D); Carmen Vanessa Castañeda-Campoy ${ }^{2}$ (D); Guadalupe Domínguez-Ramírez ${ }^{3}$ (D).

1. Instituto Tecnológico de Sonora, México. lizeth.armenta@itson.edu.mx.

2. Instituto Tecnológico de Sonora, México. carmen.castaneda@potros.itson.edu.mx

3. Instituto Tecnológico de Sonora, México. guadalupe.dominguez@potros.itson.edu.mx

*Correspondencia del autor: Lizeth Armenta Zazueta, email: lizeth.armenta@itson.edu.mx

\section{RESUMEN}

El desarrollo de las TIC en el siglo XXI ha sido un proceso de cambios vertiginosos, impulsando a los gobiernos a implementar políticas públicas que permitan a las sociedades insertarse en la sociedad de la información y del conocimiento. El objetivo es describir las realidades y retos del docente de nivel primaria en el uso de la tecnología como herramienta para la enseñanza en tiempos de contingencia, mediante el método de investigación cualitativo fenomenológico, para comprender la experiencia del docente en la modalidad educativa a distancia. Este estudio se desarrolla bajo la perspectiva teórica-metodológica del paradigma interpretativo. Es una investigación de corte cualitativa enfocada al diseño fenomenológico. Se concluye en que el reto más significativo del trabajo desde casa es lograr la colaboración entre el padre de familia, alumno y docente.

Palabras clave: Docencia; educación a distancia; aprendizaje en línea.

Cómo citar:

Armenta-Zazueta, Lizeth; Castañeda-Campoy, Carmen Vanessa; DomínguezRamírez, Guadalupe. (2021). Retos y realidades del docente de primaria en el uso de la tecnología como herramienta de enseñanza en tiempos de contingencia. Revista de Investigaciones Universidad del Quindio, 33(2), 187-200. https://doi.org/10.33975/ riuq.vol33n2.767

Información del artículo: 


\begin{abstract}
The development of ICT in the 21 st century has been a process of dizzying changes, prompting governments to implement public policies that allow societies to insert themselves into the information and knowledge society. The objective is to describe the realities and challenges of the elementary school teacher in the use of technology as a teaching tool in contingency times, through the phenomenological qualitative research method, to understand the teacher's experience in distance education. This study is developed under the theoretical-methodological perspective of the interpretive paradigm. It is qualitative research focused on phenomenological design. It is concluded that the most significant challenge of working from home is to achieve collaboration between the parent, student and teacher.
\end{abstract}

Keywords: Teaching; distance education; online learning.

\section{ANTECEDENTES}

EnMéxicoafinales demarzo del2020 se estableció una emergencia sanitaria frente a la epidemia generada por la enfermedad COVID-19, la cual se extendió hasta finales de mayo. Durante este periodo se determinaron actividades económicas esenciales y no esenciales por lo que millones de personas dejaron de asistir a sus trabajos, se adaptaron a la modalidad de teletrabajo o bien, se enfrentaron a recortes en salarios o despidos. Posterior a la fase de emergencia sanitaria, como seguimiento a la evolución de la pandemia, se establecieron semáforos del color rojo al verde. El nivel máximo de alerta por contagios se refleja con el rojo y el verde indicando el nivel más bajo de alerta. (Organización Internacional del Trabajo, 2020).

La pandemia COVID-19 ha generado un gran impacto en distintos rubros del sistema social, afectando el ámbito sanitario, social y económico, y cobrando un alto número de vidas humanas en todo el mundo. Varios países de América Latina cuentan con sistemas de salud que no están preparados para manejar una crisis sanitaria de esta magnitud. Esto afecta especialmente y de manera grave a zonas rurales y remotas, afectando particularmente a los pueblos indígenas (ONU, 2020).

En lo concerniente al ámbito educativo, esta emergencia dio lugar al cierre de actividades presenciales en todas las instituciones educativas de más de 190 países, con el fin de mitigar el impacto del virus y su propagación. Lo anterior, dio origen a tres campos principales de actuación: la adopción de modalidades de aprendizaje a distancia (con o sin uso de tecnología); el apoyo y la movilización del personal y las comunidades educativas y la atención a la salud y el bienestar integral de las y los estudiantes (CEPALUNESCO, 2020)

En México, una de las principales acciones realizadas fue la creación de la plataforma virtual Aprende en casa con el objetivo de servir como apoyo a los docentes en el desarrollo de la educación a distancia. Así mismo, la plataforma virtual brindó apoyo a alumnos y padres de familia, siendo estos últimos quienes de alguna manera quedaron al frente de la educación de sus hijos. La plataforma plantea tareas mínimas para cada nivel y grado educativo, priorizando cuatro áreas de conocimiento: Pensamiento lógico-matemático, Lenguaje y Comunicación, Convivencia Sana, Civismo y Cuidado de Salud, complementadas con actividades de lectura, videos y actividades lúdicas (Navarrete, Manzanilla y Ocaña, 2020).

Navarrete, Manzanilla y Ocaña (2020), expresan que, ante el escenario de aislamiento social, los docentes están en una situación de desventaja 
para afrontar las condiciones impuestas por la pandemia. Las medidas tomadas por la autoridad educativa para evitar la pérdida de horas clase no consideraron la opinión de los docentes, quienes, para cumplir con las indicaciones de sus superiores, optaron por enviar tareas a sus alumnos para intentar cubrir el programa de estudios buscando mantener el ritmo de trabajo habitual, resolver las dudas que hayan surgido durante la cuarentena y dar espacio dentro del quehacer pedagógico a clases especiales para hablar con los alumnos sobre el Covid-19 y las medidas sanitarias conducentes.

Por lo anteriormente planteado, la experiencia de desarrollar un ciclo escolar modalidad virtual ha significado para muchos un enorme reto a enfrentar, teniendo que capacitarse y prepararse en el ámbito tecnológico a la par que realizan su trabajo habitual como docentes: planeaciones, evaluaciones, desarrollo de recursos educativos, entre otros. Pero, todo esto solo es posible si el docente, en primer lugar, conoce y domina las TIC, tarea que por muchos años se ha querido desarrollar mediante distintos programas gubernamentales. Padilla (2018), en un arduo trabajo de investigación donde cita los programas referentes al desarrollo de la tecnología en la educación de los últimos años, donde indica que los programas de implementación no han sido insuficientes, porque o bien los softwares se vuelven obsoletos o porque se originan nuevas tecnologías que desplazan las anteriores.

El papel del docente, es de suma importancia en el proceso de aprendizaje de los alumnos, al comenzar a involucrarse con la tecnología. También es importante que el maestro, como mediadores entre el conocimiento y el alumno, comiencen a utilizarla como herramienta dentro de su labor, promoviendo el uso de medios técnicos y tecnológicos como recurso didáctico para el desarrollo de las actividades de aprendizaje.

En efecto, la utilización de las TIC dentro de las instituciones educativas debe ser cuidada, ya que no se trata solamente de usarla sino de seleccionar realmente las actividades, los formatos y recursos pertinentes para lo que se quiere lograr. No es suficiente solamente mostrar la información en diapositivas interactivas, cuando eso mismo ocurre con un pizarrón. Debe usarse la creatividad para saber de qué manera se aprovechará todo lo que la tecnología puede brindar.

Por su parte, Pedró (2017), expresa que, con un profesorado competente, el uso de la tecnología en educación permite crear entornos de enseñanza y aprendizaje que facilitan el desarrollo de las competencias que la sociedad y la economía esperan hoy de los estudiantes. Deja en claro que el potencial de la tecnología no es solamente referente a la alfabetización digital, sino como apoyo para mejorar el desempeño educativo de los estudiantes en todos los dominios.

Por esto, es que se hace necesaria la realización de diagnósticos que reflejen la realidad actual de los educadores de Educación Básica en las distintas dimensiones de las competencias TIC ya que a partir de estos reportes es posible examinar las políticas actuales y proponer lineamientos de formación permanente que incentiven el mejoramiento, actualización y consolidación del conocimiento y aplicación de la tecnología por parte de los docentes (Hernández, Arévalo, y Gamboa, 2016).

Al conocer los resultados de los diagnósticos es posible tener un panorama más realista acerca de lo que se ha logrado hasta el momento y dónde radican las deficiencias en materia de tecnología. Ante la realidad expuesta es posible cuestionar diversos aspectos: ¿Cuáles son las habilidades tecnológicas más necesarias en educación básica? ¿Qué herramientas son las que más dificultad tiene el docente en su uso? ¿En qué áreas de las TIC se consideran competentes los maestros de Educación Básica? entre muchas otras que dan una orientación a nuevas investigaciones para ahondar en el tema. 
En la situación que se vive actualmente referente a la pandemia originada por el virus SARS-CoV-2 (COVID-19), el cierre de las escuelas ha tenido un alto impacto en la educación, más de 35 millones de estudiantes y dos millones de docentes se vieron súbitamente privados del espacio escolar. En la nueva circunstancia, maestros y alumnos tienen que comunicarse desde sus respectivos hogares, en vez de encontrarse en la escuela (Mancera, Serna y Barrios, 2020).

En las décadas recientes, las tecnologías de la información y la comunicación han ganado un lugar cada vez más relevante en la educación a distancia. Mancera, Serna y Barrios (2020), expresan que las TIC tienen ventajas sobre otros medios porque ofrece comunicación instantánea, en audio, video y datos, permiten el acceso a contenidos ilimitados, grupos virtuales, diversas herramientas y el procesamiento remoto de datos.

\section{PLANTEAMIENTO DEL PROBLEMA}

En la actualidad el mundo entero se enfrenta a una situación de la cual han derivado grandes retos y que ha replanteado la forma en la que vivimos día a día. Según datos de la Organización Mundial de la Salud (OMS, 2020) la pandemia de Coronavirus SARS (COVID-19) originada en Wuhan China en Diciembre del 2019 ha afectado al día de hoy a más de 36 millones de personas en todo el mundo, con más de un millón de muertes registradas. Según Hernández (2020) en México, se confirmó el primer caso el 27 de febrero de 2020. A partir de allí comenzaron a tomarse diversas medidas para evitar su propagación, afectando así a diferentes ámbitos; entre ellos el educativo.

Álvarez(2020) menciona que de manera temprana y en atención a las recomendaciones y medidas implementadas por la OMS para contener las afectaciones de este virus, las Secretarías de Salud y de Educación Pública del Gobierno de México presentaron ante la autoridad educativa de cada uno de los estados de la República, las medidas de prevención y atención prioritarias.
Se implantó pronto el distanciamiento social lo que implicó la cancelación de las clases presenciales en todos los niveles educativos, afectando así principalmente a la educación básica. La principal estrategia empleada por la Secretaría de Educación Pública (SEP) fue la de dar continuidad a los programas curriculares desde casa mediante el uso de las tecnologías y las telecomunicaciones.

Según el Instituto Nacional de Estadística y Geografía durante 2019 en alrededor de 43\% de los hogares mexicanos se carecía de algún tipo de conexión a internet. En el mismo año, 43\% de la población de seis años o más era usuario de computadora, $70 \%$ de internet y $75 \%$ de telefonía móvil. Pero hablando de zonas rurales, sólo 22\% de la población de seis años o más que residía en ella era usuario de computadora, $47 \%$ de internet y $58.5 \%$ de telefonía celular. De quienes son usuarios de computadora en las áreas rurales, más de $47 \%$ no tiene acceso a ella en el hogar.

En el Estado Mundial de la Infancia (UNICEF, 2017) se manifiesta que, para acelerar el aprendizaje, las tecnologías de la información y la comunicación tienen que estar respaldadas en los sistemas educativos por un apoyo a la capacitación para docentes y una pedagogía firme. Este mensaje plantea la necesidad de tener al docente al frente de los aprendizajes pues no es suficiente con que los alumnos sepan utilizar una computadora, tablet o celular.

En México si bien han existido esfuerzos por acercar al docente al uso de las tecnologías, este ha sido insuficiente. Encontrándose, muchas veces, ante el conformismo o la idea de que los cambios no son buenos y el miedo ante lo desconocido y el adaptarse a los tiempos presentes.

Es así como ante esta situación, los docentes han dejado su enseñanza tradicional, para ser usuarios de las herramientas tecnológicas y poder así interactuar con sus alumnos al tiempo que tienen que afrontar los retos que conllevan las tecnologías, y que es aún mayor 
en los docentes veteranos (Sánchez, Martínez, Torres, De Agüero, Hernández, Benavides, Jaimes y Rendón, 2020). Han sido forzados a adaptarse, utilizando la tecnología al mismo que realizan todas las tareas habituales de su labor docente. Planeaciones, selección de contenidos, investigación, elección de estrategias, realización de material, evaluación de los aprendizajes. Así mismo, Sánchez, et. al (2020) expresan que desgraciadamente, las escuelas que pueden ofrecer una experiencia académica virtual completa no son muchas. La realidad es que la mayoría no están preparadas para este cambio. Se ha apremiado a docentes a hacer uso de diferentes plataformas y servicios digitales para continuidad de sus labores, apoyándose con los medios con los que cuenta.

El objetivo general de este estudio es describir las realidades y retos del docente de nivel primaria en el uso de la tecnología como herramienta para la enseñanza en tiempos de contingencia mediante el método de investigación cualitativo fenomenológico, para comprender la experiencia del docente en la modalidad educativa a distancia.

\section{Objetivos específicos}

1. Describir la realidad del docente respecto al uso de la tecnología como herramienta de enseñanza en la modalidad educativa a distancia.

2. Identificar los retos que el docente de nivel primaria enfrenta en el proceso de enseñanza en tiempos de contingencia.

\section{MARCO TEÓRICO}

\section{Sistema Educativo Mexicano}

En territorio Mexicano, la Secretaríade Educación Pública (SEP) es la encargada de coordinar las políticas públicas en materia educativa. $\mathrm{Su}$ propósito esencial es crear las condiciones que aseguren a los mexicanos y mexicanas, el acceso a una educación de excelencia, con equidad, universalidad e integralidad (SEP, 2020).
En el Artículo $3^{\circ}$ (2019) de la Constitución Política de los Estados Unidos Mexicanos se establece que toda persona tiene derecho a la educación y que es obligación del Estado garantizarlo desde el nivel inicial hasta el superior. Además de obligatoria, la educación es de carácter universal, inclusiva, pública, gratuita y laica. Este artículo reconoce que los maestros y maestras son los agentes fundamentales dentro del proceso educativo, resaltando su contribución a la transformación social. Por lo anterior tienen derecho de acceder a un sistema de formación que los capacite y actualice para contribuir y cumplir con los propósitos y objetivos del Sistema Educativo Nacional.

Desde la primera vez que se dictó este artículo en 1917, la educación ha tenido una concepción humanista, viendo a la educación como un proceso que desarrolla armónicamente todas las facultades del ser humano. No obstante, en la práctica educativa la legislación y políticas públicas han direccionado los esfuerzos a la parte intelectual, académica y en la formación para la vida productiva (Marúm, 2019).

Actualmente, el sistema educativo está retomando con especial énfasis el sentido humanista de la educación integrando elementos imprescindibles como las artes y el deporte. Esto ha resultado un gran reto para la actual Reforma Educativa pues se ha necesitado desarrollar una reestructuración de la educación obligatoria y una transformación de los modelos educativos y académicos (Márum, 2019).

\section{Formación docente en TIC}

Cuando se habla de la formación docente en las nuevas tecnologías como lo son las TIC, nos referimos al mejoramiento de la calidad y el rendimiento educativo, utilizándose como una forma de consumir y aprovechar conocimiento, siendo herramientas para enriquecer, generar y crear. Además, hay que reconocer la importancia del papel que han tenido las TIC en el desarrollo de contenidos educativos digitales que pueden 
manipularse y trabajarse en computadoras, laptops, tabletas y teléfonos celulares inteligentes (RELPE, 2015 citado por Castellanos).

En la actualidad, para preparar a los estudiantes es necesario el uso de la TIC en las instituciones educativas, así mismo también puede tener un profundo impacto en la manera en que los estudiantes aprenden. Debido a la situación derivada del confinamiento por la pandemia, se ha creado una brecha digital, por lo que se hace necesario la impartición del proceso de enseñanza - aprendizaje a través de plataformas y medios digitales accesibles para la comunidad educativa.

Por lo anterior se denota la importancia de recrear los modelos de formación y capacitación para los profesionales de la educación, Gros y Silva (2017) exponen que es necesario cambiar el antiguo modelo formativo, actualizarlo e incorporar las nuevas herramientas tecnológicas para capacitarlo como un profesional que sea más un diseñador del conocimiento y de entornos de aprendizaje, que un mero transmisor de aprendizaje.

En relación a lo antes mencionado (Izarra, Hirsch y Rodríguez, 2020) exponen que existen docentes que conocen muy poco acerca de cómo emplear y manipular las TIC, no saben aprovechar las habilidades digitales para búsqueda de información y para la creación de recursos y materiales educativos, es decir, no saben utilizar las TIC en la enseñanza, además de que muestran una limitada participación y conocimiento para su correcta manipulación.

Aunado a lo anterior el proceso educativo de enseñanza-aprendizaje en el aula con el uso de las TIC requiere de las competencias que el docente debe adquirir, aprovechando de esta manera las herramientas tecnológicas a través de las capacitaciones para afrontar los actuales retos educativos en el contexto, el principal rol del docente es el requerir de un cambio en sus prácticas metodológicas y a su vez un cambio mentalidad de sus creencias para lograr un aprendizaje significativo.

La formación docente tiene el propósito de brindar una orientación al docente para ayudar a cubrir los requerimientos necesarios para su correcta formación, por lo que la introducción de las nuevas tecnologías en su contexto profesional es de suma importancia para cumplir sus objetivos de aprendizaje (Cruz, 2020).

\section{Alfabetización tecnológica}

En la actualidad las Tecnologías de la Información y la Comunicación (TIC) en el contexto educativo son objeto de análisis debido a la importancia que implica que un docente tenga las competencias necesarias para hacer frente a este cambio tecnológico, es decir, que esté capacitado para su uso, manejo y posterior implementación didáctica en el aula. Esto supone ser un docente competente del siglo XXI (Cruz, 2019).

En los últimos años los avances en el área de la ciencia y la tecnología ha tenido un gran impacto en la sociedad, dando paso a un nuevo sistema de competencias y currículo escolar para promover el uso y apropiación de la tecnología como principal herramienta en la sociedad actual. González, Olarte y Corredor (2017) mencionan que el desarrollo de dichas competencias requiere un cambio en los ambientes de aprendizaje tradicionales y resultando necesario el contar con una metodología de enseñanza que permita desarrollar efectivamente dichas competencias, más allá de cambios secundarios en el discurso y las actividades de clase.

Es de suma importancia tener como prioridad, la capacitación y una incesante actualización de los docentes en el manejo y uso de las TICS, ya que serán las herramientas que faciliten su labor educativa. En ese sentido puede considerarse que las TIC pueden asumir el papel de apoyos didácticos, haciendo más amena e interesante la adquisición de conocimientos. 
Como se expuso anteriormente, el uso de las TIC es una herramienta indispensable para desarrollar la práctica docente, ampliando la extensión de la educación. Cruz (2019) menciona que el profesional docente que alcance las competencias tecnológicas tiene la oportunidad de innovar su enseñanza tradicional, generando cambios de estrategias didácticas efectivas, didácticas y novedosas. Una de estas estrategias es el trabajo en espacios asincrónicos, los cuales se desarrollan en un contexto novedoso que permite a los estudiantes disfrutar de asistir a las aulas virtuales, garantizando el acceso a los estudios para la adquisición de habilidades y destrezas y rompiendo las limitaciones espacio - temporales.

Para asegurar la alfabetización es necesario mantenerse en constante capacitación sobre ella, según Prieto (2016) se requiere desarrollar habilidades específicas para dar pauta a la inteligencia digital, describiéndose como un conjunto de habilidades específicas, como lo son las sociales, cognitivas y emocionales, permitiendo a la sociedad adaptarse a los cambios digitales y hacerles frente a los nuevos retos.

Así mismo, en el documento Estrategia para el Fortalecimiento y la Transformación de las Escuelas Normales, se plantea un nuevo diseño curricular con un cambio en la enseñanza y el aprendizaje para lograr incorporar el uso de las TICS. En este documento, la SEP (2019) menciona la urgencia de transformar y fortalecer las Escuelas Normales para garantizar que sigan siendo el pilar de la formación de los maestros de México y que éstos, estén a la altura de los retos del siglo XXI.

Por lo anterior se reafirma que los medios y recursos pedagógicos educativos en la nueva era digital han ocasionado una revolución, cambiando los métodos tradicionales de la enseñanza a métodos novedosos y tecnológicos solventados por las TICS, ampliando de esta manera el aprendizaje, retroalimentando y reforzando así las habilidades que permitan alcanzar los objetivos educativos.

Según Leví (2015) la tecnología de la Educación se centra en las estrategias de enseñanza de carácter tecnológico, pretenden la capacitación de futuros profesionales docentes, las nuevas tecnologías se centran en el diseño de recursos tecnológicos para el proceso de enseñanza aprendizaje y su inclusión en su utilización dentro del proceso educativo.

\section{METODOLOGÍA}

Esta investigación está orientada a describir las realidades y retos del docente de primaria en el uso de la tecnología como herramienta para la enseñanza en la modalidad educativa a distancia. En este capítulo se presentan las estrategias a implementar para lograr los objetivos de investigación establecidos.

\section{Diseño de la investigación}

Este estudio se desarrolla bajo la perspectiva teórica-metodológica del paradigma interpretativo. Es una investigación de corte cualitativa enfocada al diseño fenomenológico, el cual, según Hernández, Fernández y Baptista (2014) tiene como propósito principal explorar, describir y comprender las experiencias de las personas con respecto a un fenómeno y descubrir los elementos en común de tales vivencias. En este caso, aplica debido a que el presente proyecto busca conocer e interpretar desde la subjetividad de los individuos, las problemáticas, retos y experiencias de aprendizaje que han obtenido respecto a la tecnología durante la educación remota.

\section{Participantes}

Los participantes que conforman esta investigación cualitativa a la fecha, son un total de 10 docentes que conforman la plantilla docente de una escuela nivel primaria ubicada en una zona urbana del Sur de Sonora. Los participantes comprenden la edad de entre 30 y 61 años de 
edad, siendo $60 \%$ mujeres y $40 \%$ hombres. Para la selección de la muestra se tomó la totalidad de docentes que laboran en la institución, quienes de primer momento firmaron un consentimiento informado

Así mismo se involucró a la figura directiva del plantel quien dio acceso al campo de investigación y brindó información acerca de los docentes y su preparación en materia tecnológica. Del mismo modo, se requirió la participación de tres expertos en materia de tecnología educativa e investigación educativa quienes dieron validez a los instrumentos necesarios para la investigación.

Las técnicas de muestreo utilizadas son muestras de expertos, casos de tipo y voluntario. Las muestras de expertos han sido seleccionadas debido a que se necesita la participación de expertos en el tema de tecnología educativa para apoyo en la elaboración de los instrumentos de aplicación a los docentes que serán entrevistados. Los casos de tipo comprenden el involucramiento y participación de docentes de diversos grados pertenecientes a la plantilla docente de la primaria. Y la técnica voluntaria es debido a su naturaleza, ya que el participante recibe una invitación a participar y el decide aceptar o declinar la invitación.

\section{INSTRUMENTOS}

\section{Guión de entrevista para el docente.}

Se diseñó un guión de entrevista dirigida a todos los docentes de la institución educativa, la cual consta de 11 cuestionamientos que comprenden la opinión y experiencia de los docentes en el uso de las TICS en el proceso de enseñanza, englobando desde la definición que los docentes tienen de las TICS hasta los retos a los cuales se enfrentan al utilizarlas dentro de su labor profesional.

Para la obtención de la información se diseñó un guión de entrevista semi-estructurada (ver apéndice 3) el cual se conforma por 11 cuestionamientos abiertos. Según Hernández Sampieri (2010) la entrevista se define como una reunión para conversar e intercambiar información entre una persona (el entrevistador) y otra (el entrevistado). Las entrevistas semiestructuradas, se basan en una guía de asuntos o preguntas y el entrevistador tiene la libertad de introducir preguntas adicionales para precisar conceptos u obtener mayor información sobre los temas deseados.

Se optó por esta técnica debido a que el tema a tratar intenta ahondar en la realidad y los retos que los docentes enfrentan en cuanto a la tecnología educativa, requiriéndose las perspectivas internas y profundas de los participantes. Las entrevistas fueron realizadas en salas virtuales de Google Meets a la cual los docentes ingresaron en el horario asignado previamente por el director. Esta investigación, con relación al tiempo es transversal, utilizándose para ella el periodo comprendido del 14 al 22 de Abril del 2021.

\section{Análisis de la información}

Para esta etapa de la investigación se requirió, en primer lugar, organizar la información obtenida de manera que se realizara un documento de análisis por cada participante. Posteriormente se llevó a cabo la categorización de las respuestas obteniendo un total de cinco categorías diferentes: 1) Definición de las TIC, 2) Experiencia en el uso de las TIC, 3) Uso de las TIC en la labor docente, 4) Uso de las TIC en la educación a distancia y 5) Retos de la modalidad remota de la educación. Una vez categorizadas las respuestas de cada participante, se organizaron las respuestas obtenidas de cada categoría y se pasó a describir lo expresado por los docentes participantes.

\section{RESULTADOS}

\section{Definición de las TIC}

La pregunta introductoria a la entrevista a los docentes pretendió conocer la definición que brindaban acerca de las TIC. Como respuesta 
principal se obtuvo que son herramientas básicas que permiten la comunicación con otras personas a distancia. Son todos los dispositivos con los que podemos contar para poder comunicarnos, como ejemplos mencionaron la computadora, el internet, la tablet, el teléfono y las redes sociales.

La mayoría de los entrevistados las definieron como herramientas que pueden utilizarse en pro de la educación, siendo un medio eficaz tanto para maestros y alumnos, sobre todo en la actualidad que se desarrolla la modalidad a distancia de la educación, ya que posibilita la comunicación de los docentes con los alumnos y padres de familia.

\section{Experiencia en el uso de las TIC}

Dentro de las preguntas diseñadas para la entrevista, se incluyó una que permitiera obtener información relacionada a la experiencia que los docentes tienen en el uso de la tecnología en su labor docente, conociendo un poco más acerca de sus conocimientos, acercamientos y usos dentro del ámbito tecnológico educativo.

En primer lugar, existen los docentes que han tenido poca experiencia con la tecnología educativa a lo largo de su labor docente. Conociendo la importancia de incorporarlas en las actividades escolares, pero renuentes a utilizarlas por desconocimiento o dificultad en su uso. "Es muy importante, pero en un principio a mí me tocó que no se usaba la tecnología y solamente hacíamos todo a mano. Pero ahorita en estos tiempos creo que lo más importante es la tecnología, aunque difícil para mí porque no estoy muy acostumbrada a ella".

\section{Percepción de la tecnología}

La opinión que los docentes tienen ante la tecnología dentro del ámbito educativo, es mayormente optimista, notándose que en la actualidad son conscientes de la utilidad que éstas tienen al involucrarlas en el proceso enseñanza - aprendizaje. "Es muy buena y es muy bueno el apoyo de la tecnología cuando le damos buen uso" menciona una de las entrevistadas. Mientras que otra de las maestras expresa: "Mi opinión es que siempre han sido una fuente de ayuda, nos ha ayudado muchísimo la tecnología, yo siempre he dicho que es bueno innovar."

Hay docentes que siempre han reconocido las bondades de las tecnologías dentro de su labor docente, hay quienes, por no contar con una alfabetización tecnológica adecuada, prescindían totalmente de su uso. En la actualidad, después de utilizarlas de manera obligada ante la crisis sanitaria COVID-19 hay quienes han tenido un cambio en su percepción de éstas, expresando que las TIC son herramientas muy favorables que involucrarán en su labor docente una vez empiecen las clases presenciales.

\section{Capacitación docente en TIC}

En este apartado relacionado al tipo de capacitación docente en el uso de las tecnologías ante la modalidad educativa a distancia, una de las respuestas más frecuentes fue la de la capacitación informal. Este tipo de capacitación se da mediante la propia experiencia o bien el aprendizaje autodidacta de docentes que han investigado el funcionamiento de las plataformas de su interés apoyados en muchos casos, de videos tutoriales. La maestra que imparte clases de inglés menciona que además de por razones laborales, ha aprendido a utilizar ciertas plataformas por sus hijos quienes también son estudiantes. "No, no llevé capacitación, ni cursos, pero he aprendido a usarlas por la necesidad."

Un aspecto muy importante, es que los docentes cuentan con el apoyo del directivo del plantel quien les ha dado a conocer las herramientas básicas de comunicación de videoconferencia tales como Teams de la compañía Microsoft, Meet de la compañía Google y la plataforma Zoom. Así mismo, otros docentes que conocen las herramientas tecnológicas, brindan apoyo a sus colegas maestros. "Con el apoyo de los compañeros ya puedo unirme y conectarme a las reuniones por medio del Teams y los 
programas que usan para conectarse. Todo eso lo he aprendido ahora, yo antes ni teléfono traía" expresó uno de los entrevistados.

\section{Utilidad de las TIC en la labor docente}

La incorporación de las TIC al proceso educativo aporta grandes beneficios en diversas áreas. Optimizan tiempos y recursos, así como potencian el aprendizaje innovando en estrategias y técnicas de enseñanza. En la entrevista realizada a los docentes, las respuestas ante la interrogante de cuáles son las aportaciones de las TIC en su labor docente fueron muy diversas.

Otra de las respuestas mencionadas es que la tecnología permite estar constantemente en comunicación con los padres de familia y con los alumnos. "Actualmente estamos bien por este medio ya que nosotros nos conectamos con los padres de familia, por medio de las planeaciones les mandamos actividades y así ellos nos mandan las evidencias... de otra forma no se hubiera podido realizar".

\section{Elaboración de materiales educativos}

Además de las bondades y ventajas identificadas por los docentes al utilizar la tecnología, se conocieron los usos más recurrentes que le dan a ésta en relación a la elaboración o búsqueda de materiales educativos.

El $40 \%$ de los entrevistados no utiliza la tecnología para creación de material o bien, solo lo utilizan para búsqueda básica. Una de las respuestas expresaba que desconocía el uso de la tecnología para tal fin, por lo que pide asesoría de un colega docente quien le apoya brindándole material y actividades de los temas que necesita. Otra de las respuestas dadas por un docente, menciona que regularmente toma fotografías a las actividades plasmadas en los libros de texto, las cuales posteriormente, envía a sus alumnos por medio del teléfono celular, debido a que desconoce cómo entrar a páginas web y, sobre todo, cómo se descargan archivos de la red.
Otro 30\% de los entrevistados, utiliza de manera regular ciertos programas para el diseño de materiales, o la búsqueda de éstos en internet. Usándose principalmente, las herramientas de Microsoft: Word y Powerpoint. Así como las herramientas de Google tal como el servicio de alojamiento Drive para organizar las evidencias de los alumnos. De igual modo, los docentes utilizan diversas páginas en la red, que brindan ejercicios prácticos e interactivos de diversas temáticas cuidando que se cubran las necesidades de aprendizaje de los estudiantes, un ejemplo de esto es la página Liveworksheets.

Mientras que hay docentes que solo utilizan la tecnología para actividades básicas, el 30\% de los docentes restantes tienen un dominio más elevado en cuanto a estos recursos, lo que les permite tener diversas opciones al momento de diseñar sus actividades. Una de las experiencias encontradas es la de una maestra que aprendió a utilizar la herramienta de Google: Sites, la cual permite crear un sitio web de forma sencilla apoyado de recursos como vídeos, calendarios, presentaciones, archivos adjuntos y texto. Así mismo crea vídeos, utiliza las videollamadas e incluso una pizarra virtual, siendo cosas novedosas tanto para ella como para los niños.

\section{Dinámica de enseñanza}

La labor realizada por los docentes dentro del aula de clase y los espacios físicos educativos de todo el país, fue transformada ante las nuevas circunstancias en las que de un día a otro comenzó a desarrollarse el proceso educativo. Esta transformación fue integral, y aunque de principio todos se enfrentaron a la misma situación, las distintas circunstancias en las que vive cada docente y cada grupo de estudiantes, resultaron en muy diversas formas de llevar a cabo la enseñanza a distancia.

Como primer cambio, uno de los docentes contó que lo primero que tuvieron que hacer como comunidad escolar, fue elaborar una encuesta a través de una plataforma para hacerla llegar a 
todos los alumnos. En esa encuesta se obtuvieron datos de quiénes contaban con televisor, quiénes tenían radio, celular, internet, aparatos tecnológicos y con base a esos resultados, elegir qué opción tenía cada maestro en particular para continuar con las clases. "Vimos más viable el trabajo con cuadernillos y planificaciones que elaboramos y que les hicimos llegar a los niños a través de los grupos de Whatsapp que ya estaban previamente elaborados". "Esa fue la manera en la cual nos organizamos nosotros y ha sido una dinámica con la que hemos seguido trabajando, hemos modificado las planeaciones también porque no podemos trabajar las materias en el horario en el que nosotros estábamos trabajando, ya que era una escuela de horario completo, por lo que se tiene que reducir todo ahora a tiempo de trabajos cortos, a priorizar las necesidades de los niños".

Hay docentes que se conectan dos veces por semana con sus alumnos, y complementan la comunicación por medio de Whatsapp, recibiendo evidencias o aclarando dudas. La limitante de conexión es debido a que muchas veces los niños no pueden conectarse tanto tiempo. En muchos de los casos, es necesario aplicar ciertas modificaciones en las planeaciones para asegurar que las actividades resulten sencillas de realizar. Estas adecuaciones resultan muy distintas de un maestro a otro. Otra razón por la cual se busca diseñar actividades más sencillas es debido al cansancio físico, pero sobre todo mental que, tanto padre de familia, docentes y alumnos están experimentando después de casi un año de confinamiento.

\section{Interacción con los estudiantes}

Por otro lado, la interacción con los estudiantes ha resultado muy distinta a la que se da de manera presencial. Hay docentes que ya conocían a sus alumnos cuando cambió el modo de trabajo. Sin embargo, hay otros que fueron asignados a sus grupos una vez que comenzó la labor a distancia, por lo que no conocen en persona a sus estudiantes.
El primer escenario resultante de la comunicación distante, es que los docentes se comunican de manera ocasional con los niños por medio de videollamadas, y el trato más común es a través de los padres de familia quienes instruyen a sus hijos en las actividades a realizar.

Otros usan como estrategia mandar la planeación de las actividades al iniciar la semana y recibir evidencias el día viernes. Los jueves se conectan por videollamada donde comentan acerca de las actividades realizadas en la semana, aclaran dudas y comentan todo lo aprendido. De la misma manera, el docente realiza reuniones virtuales con los padres de familia cuando se requiere.

Existen casos donde se dificulta la interacción cercana con los alumnos. Por falta de recursos, conectividad a internet e incluso, tiempo por parte del padre de familia para estar al pendiente durante las videollamadas, puesto que muchos de ellos trabajan.

Hay docentes que optaron por no comunicarse por medio de videollamadas para no abrumar al padre de familia y al alumno, optando solamente por enviar el plan de trabajo y recibir las evidencias. Esto es principalmente en apoyo y solidaridad por los padres que trabajan y poro no rezagar a los alumnos que no cuentan con la misma oportunidad de acceso a las tecnologías que otros estudiantes.

\section{Retos de la modalidad remota de la educación}

Reto 1. Concientizar al padre de familia respecto a la nueva modalidad de trabajo a distancia. Varios docentes coinciden en que uno de los retos más importantes a afrontar ante el cambio de trabajo educativo fue el acostumbrar a los padres de familia a trabajar a distancia. Fue un aspecto determinante a la hora de comenzar a implementar el nuevo plan de trabajo, puesto que a este nivel educativo los niños no son autosuficientes ni autónomos para el uso de las herramientas tecnológicas. Por ello, los docentes tuvieron que convencer a los padres de familia 
acerca del papel tan importante que ellos fungen.

Reto 2. Utilizar la tecnología a favor de la enseñanza. Para muchos docentes, migrar de la enseñanza presencial a la remota implicó enfrentarse a situaciones que los llevaron fuera de su zona de confort al adentrarse en tecnologías que tal vez no conocían o dominaban. Por ello, coincidieron en que uno de los retos más grandes fue utilizar la tecnología para continuar con su proceso de enseñanza y llevarlo del escenario presencial al virtual.

El mayor reto para uno de los docentes ha sido el utilizar forzosamente la tecnología, puesto que desconocía mayormente su uso. No obstante, es consciente que de otra forma no se hubiera podido dar la enseñanza y que de no haber sido por la tecnología no hubiera podido comunicarse con los padres de familia y los alumnos.

Reto 3. Adaptación de la labor docente. Continuando con los retos enfrentados por los docentes, uno de ellos fue el adaptar y modificar su forma de trabajo, procurando incluir las herramientas que tanto ellos como sus alumnos tenían al alcance. Al comenzar a usar nuevas herramientas tecnológicas, debían procurar conocerlas lo más posible, para poder instruir a los padres de familia quienes también las usarían, teniendo que investigar a fondo para poder comunicarles qué van a hacer y cómo van a hacerlo. Aunado a esto, algunos maestros no conocen a sus alumnos, por lo que resulta más desafiante el elegir las actividades apropiadas a sus características y estilos de aprendizaje.

Reto 4. Falta de disposición, interés y motivación de los padres de familia y alumnos. Diversos docentes coincidieron en que uno de los retos más significativos ha sido la disposición que tiene el padre de la familia. Uno de los docentes, comentó que algunos padres de familia no estaban al pendiente cuando intentaba entablar comunicación con ellos. No le mandan los trabajos, ni contestan sus llamadas telefónicas. Menciona que hay muchos padres con los que no ha logrado una comunicación muy buena o eficiente. Ese es el mayor de los retos, el que se adapten a esta modalidad.

\section{CONCLUSIONES}

El principal propósito al realizar esta investigación fue el rescatar las perspectivas, los retos y realidades que los docentes de educación básica tienen acerca del uso de la tecnología en la educación en los tiempos contemporáneos donde se ha visto la necesidad de establecer la educación virtual / remota como el principal medio de enseñanza aprendizaje.

En cuanto a los usos que los docentes han realizado a lo largo de su práctica profesional y en la actualidad, ante los cambios de modalidad educativa, las principales aplicaciones de la tecnología habían sido en relación a generar diversidad de contenidos educativos incluyendo la didáctica e interactividad. Los usos que la tecnología ha protagonizado hoy en día son más relacionados a la inclusión de nuevas formas de trabajo que involucran la comunicación sincrónica y asincrónica.

La primera es evidente en el uso de las salas virtuales que permiten la interacción en tiempo real a través de video llamadas, complementándose con el uso de plataformas asincrónicas como el correo electrónico, entre otras.

Uno de los resultados más significativos de este trabajo es el conocer la perspectiva del docente en cuanto a los retos que ha enfrentado al incrementar el uso de las tecnologías en su labor profesional, así como el concientizar al padre de familia respecto a esta nueva modalidad, buscando de esta manera motivar tanto al niño como a los padres para trabajar en colaboración, para el logro de los aprendizajes. Así mismo, otro de los retos es el adaptar la labor docente de presencial a virtual, trayendo consigo necesidades de capacitación en muchas de las áreas tecnológicas para poder brindar una 
educación de calidad, así como el desafío que nace de la dificultad de acceso a la tecnología con la que se cuenta en los hogares de muchos estudiantes a lo largo del territorio mexicano.

En este proyecto se identificaron áreas de oportunidad, Por ello, se presentan algunas recomendaciones, tales como, continuar con la capacitación a docentes acerca de herramientas tecnológicas que puedan ser de utilidad en el proceso de enseñanza aprendizaje, así como el requerir a un profesional experto en tecnología que cuente con las habilidades y competencias tecnológicas para brindar orientación y apoyo a los docentes. De la misma manera es preciso que el docente busque siempre realizar un diagnóstico tecnológico a sus estudiantes, para tener conocimiento de las necesidades en materia tecnológica y tomarlo de referencia para diseñar sus planes de clase.

\section{REFERENCIAS}

1. Álvarez, G. (2020) Covid-19. Cambiar de paradigma educativo. Departamento de Investigaciones Educativas del Cinvestav. http://www.comie.org.mx/v5/sitio/2020/04/16/covid-19-cambiar-deparadigma-educativo/

2. Castellanos, M. (2015) ¿Son las TIC realmente, una herramienta valiosa para fomentar la calidad de la educación?. Laboratorio Latinoamericano de Evaluación de la Calidad de la Educación. Working paper (2) https://unesdoc.unesco.org/ark:/48223/pf0000244952.locale=es

3. Comisión Económica Para América Latina-Organización de las Naciones Unidas Para la Educación, la Ciencia y la Cultura (2020). La educación en tiempos de la pandemia de COVID-19. Informe COVID-19 CEPAL-UNESCO. https://repositorio.cepal.org/bitstream/handle/11362/45904/1/ S2000510_es.pdf

4. Cruz, M. (2020). Formación continua del docente como factor de la calidad educativa universitaria. Revista Científica Internacional 3(1) 73-79. https://doi.org/10.46734/revcientifica.v3i1.21.

5. Cruz, E. (2019). Importancia del manejo de competencias tecnológicas en las prácticas docentes de la Universidad Nacional Experimental de la Seguridad (UNES). Revista Educación. 43 (1). Venezuela. https://www.redalyc.org/jatsRepo/440/44057415013/html/index.html

6. González, D., Olarte, F. y Corredor, J. (2017). La alfabetización tecnológica: de la informática al desarrollo de competencias tecnológicas. Estudios pedagógicos (Valdivia). 43(1). 193-212. https:// dx.doi.org/10.4067/S0718-07052017000100012

7. Gros, B. y Silva, J. (2017). La formación del profesorado como docente en los espacios virtuales de aprendizaje. Revista Iberoamericana de Educación (ISSN: 1681-5653). https://rieoei.org/ historico/deloslectores/959Gros.PDF

8. Hernández, R., Fernández, C., y Baptista, P. (2014). Metodología de la investigación: (6a. ed.). México D.F.: McGraw-Hill.

9. Hernández R. (2020) COVID-19 en México: casos por entidad y cronología del coronavirus. La razón de México. https://www.razon.com.mx/mexico/covid-19-mexico-cuantos-casos-entidadcronologia-coronavirus-muertos-estado-81909

10. Hernández, C., Arévalo, M. y Gamboa, A. (2016). Competencias TIC para el desarrollo profesional docente en educación básica. Praxis \& Saber, vol. 7. núm. 14. https://doi. org $/ 10.19053 / 22160159.5217$

11. Instituto Nacional de Estadística y Geografía (2019) Encuesta Nacional sobre Disponibilidad y Uso de Tecnologías de la Información en los Hogares. https://www.inegi.org.mx/programas/ dutih/2019/default.html\#Tabulados

12. Izarra, D., Hirsch Adler, A., y Rodríguez León, M. (2020). Profesorado de posgrado y el desarrollo del pensamiento crítico. Innovación Educativa, vol. 20, número 83, 166. https://www.593dp.com/ index.php/593_Digital_Publisher/article/view/426/611

13. Leví, G. (2015). CAC̄HEIRO GONZÁLEZ, M. L. (coord.) (2014). Educación y tecnología: estrategias didácticas para la integración de las TIC. Madrid, UNED, 257 páginas. Bordón. Revista De Pedagogía, 67(2), 160-161 https://recyt.fecyt.es/index.php/BORDON/article/view/31856

14. Mancera, C., Serna, L. y Barrios, M. (2020). Pandemia: maestros, tecnología y desigualdad. https:// educacion.nexos.com.mx/?p=2286 
15. Marúm, E. (2019). Estado de la situación educativa en México y propuestas de mejora a raíz de la Reforma Educativa. Organismo para la Mejora Continua de la Educación. Propuestas de los candidatos a integrar la Junta Directiva. Revista mexicana de investigación educativa. 24(83), 1135-1219. https://www.redalyc.org/jatsRepo/140/14063077008/html/index.html

16. Navarrete, Z., Manzanilla, H. y Ocaña, L. (2020). Políticas implementadas por el gobierno mexicano frente al COVID-19. El caso de la educación básica. Revista Latinoamericana de Estudios Educativos (México), vol. L, núm. Esp.-, pp. 143-172. https://doi.org/10.48102/ rlee.2020.50.ESPECIAL.100

17. Organización Mundial de la Salud (2020) Naciones Unidas México. Información Oficial de las Naciones Unidas. https://coronavirus.onu.org.mx/

18. Organización de las Naciones Unidas. (2020). Informe: El impacto del COVID-19 en América Latina y el Caribe. https://peru.un.org/sites/default/files/2020-07/SG\%20Policy\%20brief\%20 COVID\%20LAC\%20\%28Spanish\%29_10\%20July_0.pdf

19. Organización Internacional del Trabajo (2020). Panorama Laboral en tiempos de la COVID-19. https://www.ilo.org/wcmsp5/groups/public/---americas/---ro-lima/---ilo-mexico/documents/ publication/wcms_757364.pdf

20. Padilla, S. (2018). Usos y actitudes de los formadores de docentes ante las TIC. Entre lo recomendable y la realidad de las aulas. Apertura vol. 10 no. 1. https://doi.org/10.32870/ap.v10n1.1107

21. Pedró F. (2017) Tecnologías para la transformación de la educación. Fundación Santillana. Spain.

22. Prieto, P. (2016). ¿Se potencia la inteligencia digital en las escuelas?. [Blog] La servilleta. https:// www.pacoprieto.com/se-potencia-la-inteligencia-digital-en-las-escuelas/

23. Sánchez, M., Martínez A., Torres, R., De Agüero, M., Hernández, A., Benavides, M., Jaimes, C. y Rendón, V. (2020). Retos educativos durante la pandemia de COVID-19: una encuesta a profesores de la UNAM. Revista Digital Universitaria, 21(3).

24. Secretaría de Educación Pública (2019) Estrategia Nacional de Mejora de las Escuelas Normales. Acuerdo Educativo Nacional Implementación operativa. http:/gaceta.diputados.gob.mx/ Gaceta/64/2019/nov/MejEscNormales.pdf

25. Secretaría de Educación Pública (2020) Visión y Misión de la SEP. https://www.gob.mx/sep/ acciones-y-programas/vision-y-mision-de-la-sep?state=published

26. Fondo de las Naciones Unidas Para la Infancia (2017) Estado Mundial de la Infancia 2017. Niños en un mundo digital (UNICEF) https://www.unicef.org/media/48611/file 